\title{
Heterogeneous Ozonolysis of Squalene: Gas-Phase Products Depend on Water Vapor Concentration
}

Caleb Arata*£§, Nadja Heine ${ }^{\ddagger}$, Nijing Wang ${ }^{\llbracket}$, Pawel K. Misztal ${ }^{\S \#}$, Pawel Wargocki^${ }^{\wedge}$, Gabriel Bekö $^{\wedge}$, Jonathan Williams ${ }^{\llbracket}$, William W Nazaroff ${ }^{\|}$, Kevin R. Wilson ${ }^{\ddagger}$, Allen H. Goldstein ${ }^{\S \|}$

${ }^{\dagger}$ Department of Chemistry, ${ }^{\S}$ Department of Environmental Science, Policy and Management, and "Department of Civil and Environmental Engineering, University of California, Berkeley, California 94720 United States

${ }^{\ddagger}$ Chemical Sciences Division, Lawrence Berkeley National Laboratory, Berkeley, California 94720, United States

`Air Chemistry Department, Max Planck Institute for Chemistry, 55128 Mainz, Germany

${ }^{\wedge}$ Department of Civil Engineering, Technical University of Denmark, 2800 Kgs. Lyngby, Denmark

\# Now at: Department of Civil, Architectural and Environmental Engineering, The University of Texas at Austin, Austin, TX 78712

*Email: caleb.arata@berkeley.edu

\section{Supporting Information}

Pages S1-S8

Tables S1-S5

Figures S1-S4 
Table S1: Geranyl acetone concentration data used to calculate production from ozonolysis. Once concentrations were stable in the flow tube reactor, a 'Raw' measurement was taken. Shortly after, the particle flow was replaced with a flow of $\mathrm{N}_{2}$, and squalene particles were removed from the flow tube reactor, allowing for a background measurement. Background measurements were taken 2 min after the particle flow was removed, when $95 \%$ of particles are removed from the flow tube.

\begin{tabular}{|c|c|c|c|c|}
\hline $\mathrm{RH}(\%)$ & $\mathrm{O}_{3}$ Exposure (ppb h) & Background $\left(\mu \mathrm{g} \mathrm{m}^{-3}\right)$ & $\operatorname{Raw}\left(\mu \mathrm{g} \mathrm{m}^{-3}\right)$ & Difference $\left(\mu \mathrm{g} \mathrm{m}^{-3}\right)$ \\
\hline 0 & 9 & $\begin{array}{ll} \\
9\end{array}$ & 14 & 5 \\
\hline 0 & 12 & 1 & 4 & 3 \\
\hline 0 & 17 & 0 & 0 & 0 \\
\hline 0 & 21 & 0 & 0 & 0 \\
\hline 0 & 27 & 0 & 0 & 0 \\
\hline 0 & 32 & 0 & 0 & 0 \\
\hline 30 & 5 & 69 & 70 & 1 \\
\hline 30 & 10 & 22 & 37 & 15 \\
\hline 30 & 12 & 3 & 10 & 7 \\
\hline 30 & 17 & 0 & 1 & 1 \\
\hline 30 & 23 & 0 & 0 & 0 \\
\hline 30 & 30 & 0 & 0 & 0 \\
\hline 50 & 6 & 149 & 160 & 11 \\
\hline 50 & 10 & 20 & 34 & 14 \\
\hline 50 & 12 & 3 & 9 & 6 \\
\hline 50 & 16 & 0 & 1 & 1 \\
\hline 50 & 21 & 0 & 0 & 0 \\
\hline 50 & 26 & 0 & 0 & 0 \\
\hline 50 & 28 & 0 & 0 & 0 \\
\hline 70 & 6 & 118 & 127 & 9 \\
\hline 70 & 9 & 24 & 43 & 19 \\
\hline 70 & 12 & 3 & 12 & 9 \\
\hline 70 & 16 & 0 & 1 & 1 \\
\hline 70 & 21 & 0 & 0 & 0 \\
\hline 70 & 31 & 0 & 0 & 0 \\
\hline
\end{tabular}


Table S2: 6-MHO concentration data used to calculate production from ozonolysis. Once concentrations were stable in the flow tube reactor, a 'Raw' measurement was taken. Shortly after, the particle flow was replaced with a flow of $\mathrm{N}_{2}$, and squalene particles were removed from the flow tube reactor, allowing for a background measurement. Background measurements were taken 2 min after the particle flow was removed, when $95 \%$ of particles are removed from the flow tube.

\begin{tabular}{crrrr}
\hline $\mathrm{RH}(\%)$ & $\mathrm{O}_{3}$ Exposure $(\mathrm{ppb} \mathrm{h})$ & Background $\left(\mu \mathrm{g} \mathrm{m}^{-3}\right)$ & Raw $\left(\mu \mathrm{g} \mathrm{m}^{-3}\right)$ & Difference $\left(\mu \mathrm{g} \mathrm{m}^{-3}\right)$ \\
\hline 0 & 9 & 36 & 94 & 57 \\
0 & 12 & 15 & 69 & 52 \\
0 & 17 & 7 & 31 & 25 \\
0 & 21 & 2 & 18 & 15 \\
0 & 27 & 1 & 7 & 6 \\
0 & 32 & 1 & 3 & 2 \\
30 & 5 & 151 & 152 & 1 \\
30 & 10 & 112 & 172 & 59 \\
30 & 12 & 51 & 132 & 79 \\
30 & 17 & 12 & 58 & 45 \\
30 & 23 & 6 & 31 & 25 \\
30 & 30 & 3 & 13 & 9 \\
50 & 6 & 290 & 401 & 115 \\
50 & 10 & 105 & 218 & 112 \\
50 & 12 & 41 & 169 & 128 \\
50 & 16 & 6 & 90 & 83 \\
50 & 21 & 2 & 47 & 45 \\
50 & 26 & 1 & 27 & 25 \\
50 & 28 & 1 & 15 & 14 \\
70 & 6 & 240 & 355 & 115 \\
70 & 9 & 109 & 260 & 151 \\
70 & 12 & 42 & 216 & 173 \\
70 & 16 & 6 & 121 & 115 \\
70 & 21 & 2 & 68 & 65 \\
70 & 31 & 2 & 12 & 11
\end{tabular}


Table S3: 4-OPA concentration data used to calculate production from ozonolysis. Once concentrations were stable in the flow tube reactor, a 'Raw' measurement was taken. Shortly after, the particle flow was replaced with a flow of $\mathrm{N}_{2}$, and squalene particles were removed from the flow tube reactor, allowing for a background measurement. Background measurements were taken 2 min after the particle flow was removed, when $95 \%$ of particles are removed from the flow tube.

\begin{tabular}{ccrrr}
\hline $\mathrm{RH}(\%)$ & $\mathrm{O}_{3}$ Exposure $(\mathrm{ppb} \mathrm{h})$ & Background $\left(\mu \mathrm{g} \mathrm{m}^{-3}\right)$ & Raw $\left(\mu \mathrm{g} \mathrm{m}^{-3}\right)$ & Difference $\left(\mu \mathrm{g} \mathrm{m}^{-3}\right)$ \\
\hline 0 & 9 & 148 & 185 & 38 \\
0 & 12 & 147 & 205 & 58 \\
0 & 17 & 134 & 209 & 75 \\
0 & 21 & 105 & 202 & 96 \\
0 & 27 & 96 & 192 & 95 \\
0 & 32 & 101 & 199 & 38 \\
30 & 5 & 191 & 229 & 19 \\
30 & 10 & 335 & 353 & 47 \\
30 & 12 & 378 & 425 & 125 \\
30 & 17 & 368 & 492 & 160 \\
30 & 23 & 344 & 502 & 184 \\
30 & 30 & 337 & 522 & 42 \\
50 & 6 & 848 & 887 & 20 \\
50 & 10 & 579 & 601 & 75 \\
50 & 12 & 712 & 785 & 240 \\
50 & 16 & 676 & 917 & 323 \\
50 & 21 & 626 & 950 & 399 \\
50 & 26 & 618 & 1020 & 398 \\
50 & 28 & 640 & 1038 & 11 \\
70 & 9 & 857 & 871 & 79 \\
70 & 12 & 1083 & 1165 & 282 \\
70 & 16 & 1233 & 1516 & 447 \\
70 & 21 & 1179 & 1630 & 606 \\
70 & 31 & 1136 & 1734 &
\end{tabular}


Table S4: 1,4-butanedial concentration data used to calculate production from ozonolysis. Once concentrations were stable in the flow tube reactor, a 'Raw' measurement was taken. Shortly after, the particle flow was replaced with a flow of $\mathrm{N}_{2}$, and squalene particles were removed from the flow tube reactor, allowing for a background measurement. Background measurements were taken 2 min after the particle flow was removed, when $95 \%$ of particles are removed from the flow tube.

\begin{tabular}{ccrrr}
\hline $\mathrm{RH}(\%)$ & $\mathrm{O}_{3}$ Exposure $(\mathrm{ppb} \mathrm{h})$ & Background $\left(\mu \mathrm{g} \mathrm{m}^{-3}\right)$ & Raw $\left(\mu \mathrm{g} \mathrm{m}^{-3}\right)$ & Difference $\left(\mu \mathrm{g} \mathrm{m}^{-3}\right)$ \\
\hline 0 & 9 & 81 & 106 & 25 \\
0 & 12 & 87 & 126 & 40 \\
0 & 17 & 88 & 143 & 55 \\
0 & 21 & 67 & 150 & 82 \\
0 & 27 & 57 & 142 & 85 \\
0 & 32 & 57 & 143 & 85 \\
30 & 5 & 66 & 87 & 20 \\
30 & 10 & 112 & 135 & 25 \\
30 & 12 & 122 & 166 & 45 \\
30 & 17 & 110 & 205 & 95 \\
30 & 23 & 100 & 218 & 135 \\
30 & 30 & 92 & 229 & 25 \\
50 & 10 & 153 & 178 & 46 \\
50 & 12 & 161 & 207 & 86 \\
50 & 16 & 152 & 238 & 96 \\
50 & 21 & 146 & 242 & 107 \\
50 & 26 & 149 & 257 & 101 \\
50 & 28 & 159 & 260 & 16 \\
70 & 9 & 189 & 206 & 23 \\
70 & 12 & 223 & 246 & 43 \\
70 & 16 & 254 & 297 & 50 \\
70 & 21 & 239 & 289 & 53 \\
70 & 31 & 257 & 309 &
\end{tabular}


Table S5: Acetone concentration data used to calculate production from ozonolysis. Once concentrations were stable in the flow tube reactor, a 'Raw' measurement was taken. Shortly after, the particle flow was replaced with a flow of $\mathrm{N}_{2}$, and squalene particles were removed from the flow tube reactor, allowing for a background measurement. Background measurements were taken 2 min after the particle flow was removed, when $95 \%$ of particles are removed from the flow tube.

\begin{tabular}{crrrr}
\hline $\mathrm{RH}(\%)$ & $\mathrm{O}_{3}$ Exposure $(\mathrm{ppb} \mathrm{h})$ & Background $\left(\mu \mathrm{g} \mathrm{m}^{-3}\right)$ & Raw $\left(\mu \mathrm{g} \mathrm{m}^{-3}\right)$ & Difference $\left(\mu \mathrm{g} \mathrm{m}^{-3}\right)$ \\
\hline 0 & 9 & 81 & 167 & 84 \\
0 & 12 & 71 & 173 & 102 \\
0 & 17 & 71 & 169 & 98 \\
0 & 21 & 57 & 165 & 107 \\
0 & 27 & 52 & 154 & 102 \\
0 & 32 & 52 & 158 & 105 \\
30 & 5 & 105 & 137 & 32 \\
30 & 10 & 142 & 227 & 84 \\
30 & 12 & 123 & 267 & 141 \\
30 & 17 & 92 & 293 & 197 \\
30 & 23 & 81 & 289 & 206 \\
30 & 30 & 75 & 288 & 213 \\
50 & 6 & 163 & 240 & 77 \\
50 & 10 & 135 & 249 & 112 \\
50 & 12 & 110 & 289 & 178 \\
50 & 16 & 71 & 301 & 230 \\
50 & 21 & 62 & 302 & 238 \\
50 & 26 & 60 & 319 & 256 \\
50 & 28 & 62 & 307 & 243 \\
70 & 6 & 137 & 216 & 77 \\
70 & 9 & 158 & 293 & 133 \\
70 & 12 & 142 & 337 & 194 \\
70 & 16 & 122 & 381 & 258 \\
70 & 21 & 149 & 428 & 278 \\
70 & 31 & 184 & 459 & 278
\end{tabular}




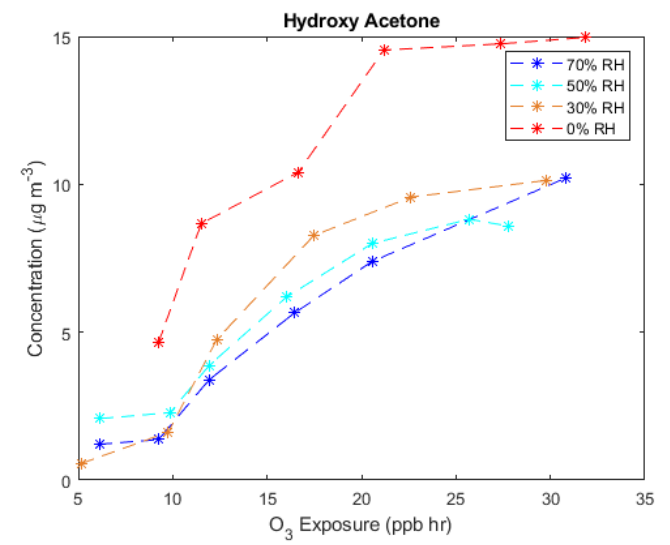

Figure S1: Hydroxy acetone concentration as a function of humidity and $\mathrm{O}_{3}$ exposure.

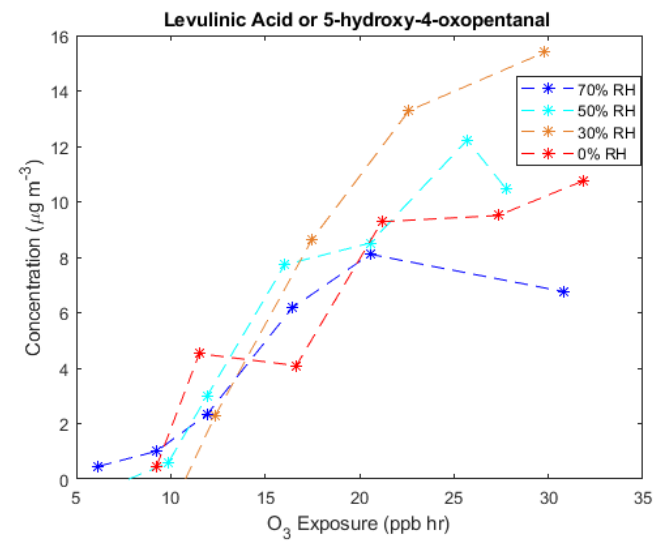

Figure S2:5-hydroxy-4-oxopentanal and levulinic acid concentration as a function of humidity and $\mathrm{O}_{3}$ exposure.

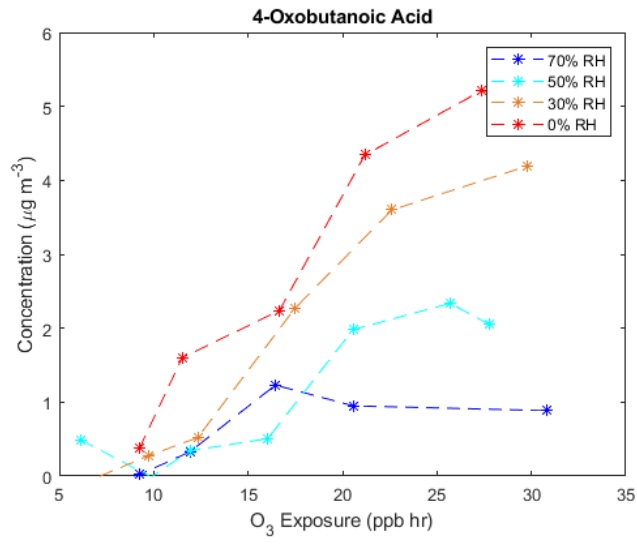

Figure S3: 4-oxopentanoic acid concentration as a function of humidity and $\mathrm{O}_{3}$ exposure. 


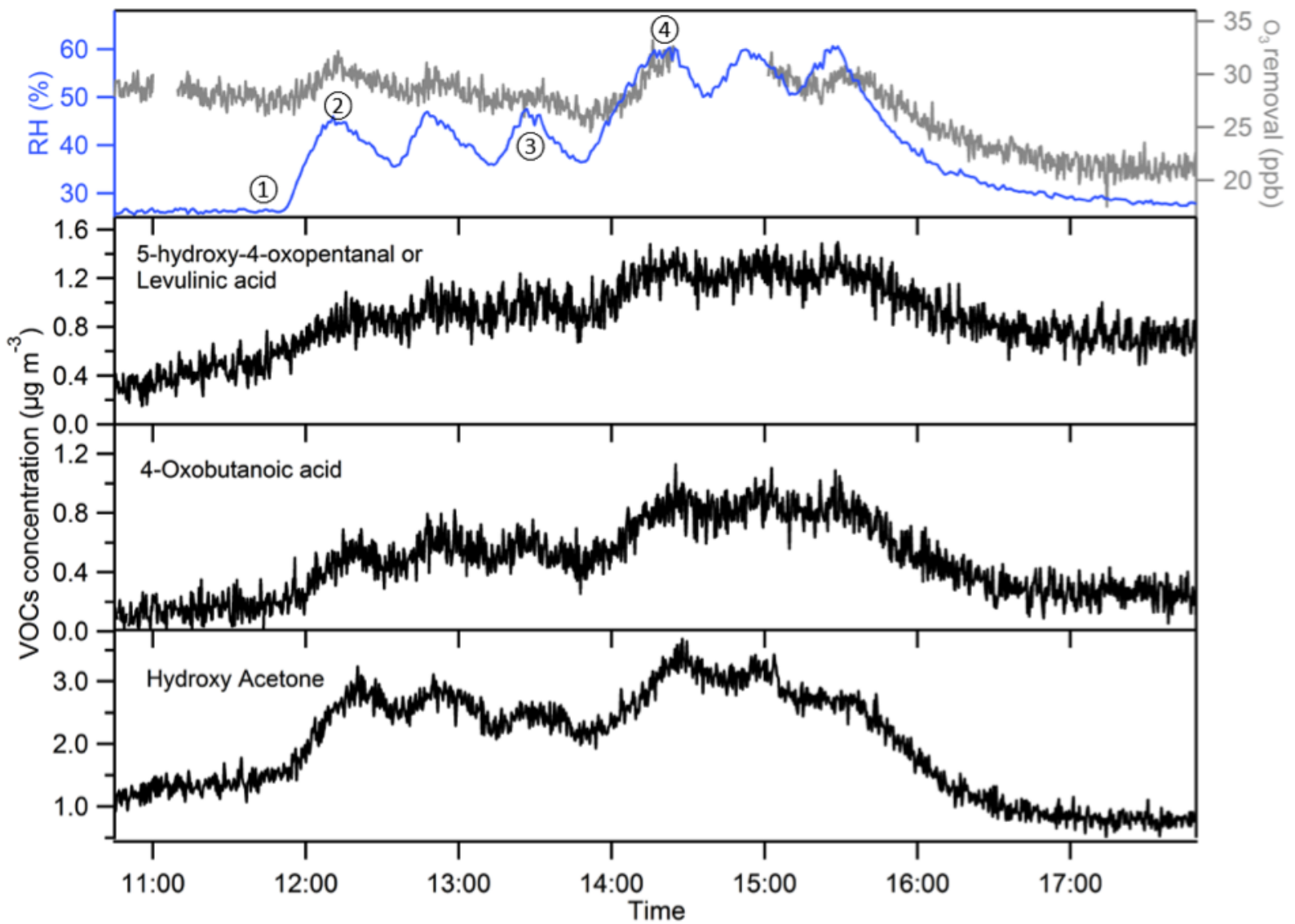

Figure S4: Time series of other squalene oxidation products with relative humidity and $\mathrm{O}_{3}$ removal (upper panel) for the clothing experiment $\left(\mathrm{O}_{3}\right.$ removal is the difference between $\mathrm{O}_{3}$ measured in supply air and the chamber; the data gap is due to the supply air measurement). 